\title{
2 MW UPGRADE OF THE FERMILAB MAIN INJECTOR*
}

\author{
W. Chou ${ }^{\#}$ for the Proton Driver Study II Group, FNAL, Batavia, IL 60510, USA
}

\section{Abstract}

In January 2002, the Fermilab Director initiated a design study for a high average power, modest energy proton facility. An intensity upgrade to Fermilab's 120$\mathrm{GeV}$ Main Injector (MI) represents an attractive concept for such a facility, which would leverage existing beam lines and experimental areas and would greatly enhance physics opportunities at Fermilab and in the U.S. With a Proton Driver replacing the present Booster, the beam intensity of the MI is expected to be increased by a factor of five. Accompanied by a shorter cycle, the beam power would reach $2 \mathrm{MW}$. This would make the MI a more powerful machine than the SNS or the J-PARC. Moreover, the high beam energy $(120 \mathrm{GeV})$ and tunable energy range $(8-120 \mathrm{GeV})$ would make it a unique high power proton facility. The upgrade study has been completed and published [1]. This paper gives a summary report.

\section{INTRODUCTION}

On January 10, 2002, the Fermilab Director issued a charge requesting a design report that would consist of three parts:

- An $8 \mathrm{GeV}$ linac based Proton Driver;

- An $8 \mathrm{GeV}$ synchrotron based Proton Driver;

- A $2 \mathrm{MW}$ upgrade of the Main Injector.

About 2/3 of the report has been completed and published [1]. This paper is a summary of the MI upgrade part. For details the readers are referred to Part B of Ref. [1].

In the present Fermilab accelerator complex, which consists of two straight machines, six rings and a number of beam lines, the bottleneck that limits the beam intensity is the Booster. It is a 30-year-old machine and has never been upgraded. The machines on both sides of the Booster - the Linac in the upstream and the Main Injector in the downstream - can deliver or receive more particles than they do now. However, the number of protons per cycle from the Booster is limited to about $5 \times 10^{12}$. Otherwise the beam loss would become prohibitive. When the Booster is replaced by a new machine, dubbed the Proton Driver, this bottleneck would be eliminated. Consequently the beam intensity in the Main Injector would be greatly increased.

\section{UPGRADE GOALS}

The goals of this upgrade study are as follows:

- Increase beam intensity by a factor of 5;

- $\quad$ Reduce cycle time by $20 \%$;

- Increase beam power by a factor of 6 .

The main parameters are listed in Table 1. As a comparison, the present parameters are also listed. The extraction energy is tunable in the range from 8 to 120 $\mathrm{GeV}$. At lower energy, the cycle time will be shorter.
Therefore, the beam power would remain about the same and only be slightly reduced due to the constant "overhead" part of the cycle (e.g., injection front porch, parabola at the beginning of the acceleration, flat top, and magnet reset at the end of the cycle, see Fig. 4 below). The MI cycle time is a multiple of the Booster cycle, which is $66.7 \mathrm{~ms}(15 \mathrm{~Hz})$. To accelerate 6 Booster batches to $120 \mathrm{GeV}$ presently takes 28 Booster cycles. This would be shortened to 23 Booster cycles in the upgrade.

Table 1: Main Injector Parameters

\begin{tabular}{|l|c|c|}
\hline & Present & Upgrade \\
\hline Injection energy $(\mathrm{GeV})$ & 8 & 8 \\
\hline Extraction energy $(\mathrm{GeV})$ & 120 & $8-120$ \\
\hline Protons per MI cycle $\left(10^{13}\right)$ & 3 & 15 \\
\hline Cycle time at $120 \mathrm{GeV}(\mathrm{s})$ & 1.867 & 1.533 \\
\hline Beam power $(\mathrm{MW})$ & 0.3 & 1.9 \\
\hline
\end{tabular}

\section{BEAM DYNAMICS ISSUES}

\section{Transition Crossing}

One main concern is possible particle loss and emittance dilution when an intense proton beam crosses the transition, which occurs at $\gamma=\gamma_{T}=21.6$. A series of simulations using the code ESME were carried out. It seemed that with a $\gamma_{\mathrm{T}}$-jump system, which will be described below, this problem can be resolved. Figure 1 shows two cases of a proton bunch after the transition, one without and another with $\gamma_{\mathrm{T}}$-jump. The latter fit well in the RF bucket and has no losses.
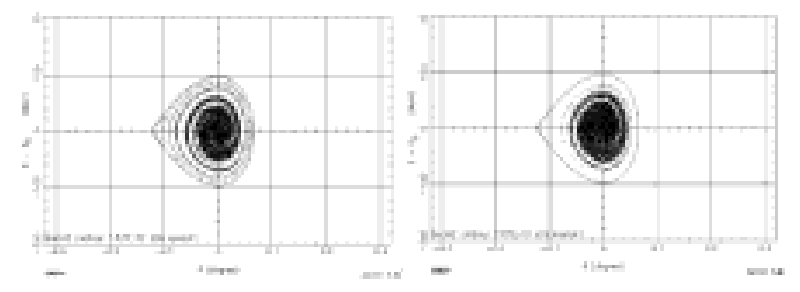

Figure 1: ESME simulation of a proton bunch in the longitudinal phase space after the transition. Left: without

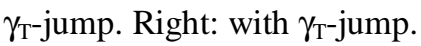

\section{Space Charge and Beam Stability}

The coherent and incoherent betatron tune shift from space charge and image charge forces throughout the cycle were computed. The single bunch longitudinal microwave instability was also calculated using the KeilSchnell criterion. The results are shown in Fig. 2. The tune shift (maximum $<-0.2$ ) is tolerable. The MI impedance budget $\mathrm{Z}_{\mid} / \mathrm{h}$ is $1.6 \mathrm{ohm}$ [2], which is below the instability threshold except during the transition crossing, which will be taken care of by $\gamma_{\mathrm{T}}$-jump. Therefore, they should not be major concerns. 

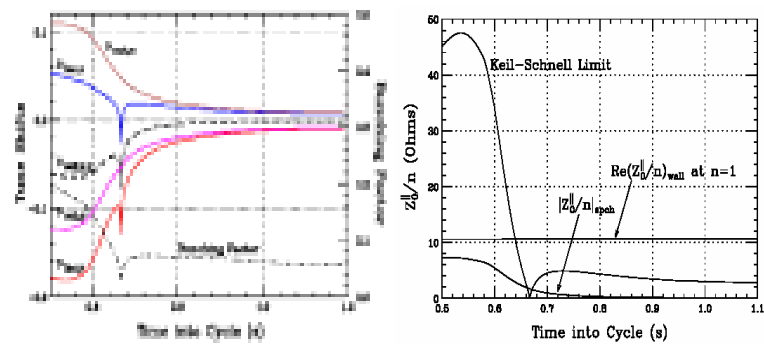

Figure 2: Left: Betatron tune shift during the cycle. Right: Instability limit during the cycle; the lowest point is at transition crossing.

\section{TECHNICAL SYSTEMS UPGRADE}

In order for the Main Injector to operate at $2 \mathrm{MW}$, most of the technical systems need to be upgraded. Some of these upgrades are major, some moderate. Here is an overview.

\section{RF System}

To accelerate 5 times more particles in a shorter period, the RF system requires a major upgrade. The number of cavities needs to be increased from 18 to 22 . The number of power amplifier of each cavity also needs to be doubled from one to two, each capable of supplying approximately $400 \mathrm{~kW}$ of peak RF power. The second power amplifier will be installed in place of the existing cavity balancing top hat capacitor, as shown in Fig. 3 .

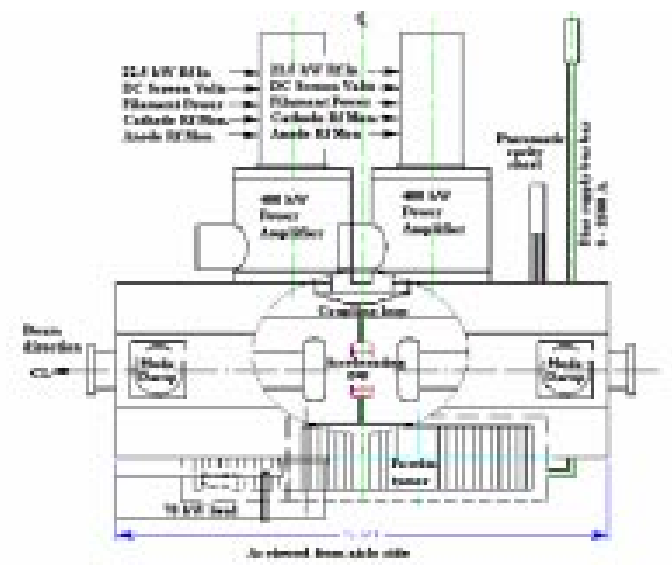

Figure 3: Twin power amplifiers on an RF cavity.

\section{Magnet}

The MI magnets will function adequately in the upgrade and modifications are unnecessary.

\section{Power Supplies}

A shorter cycle requires an increase of the maximum ramp rate from $240 \mathrm{GeV} / \mathrm{s}$ to $305 \mathrm{GeV} / \mathrm{s}$. A modest upgrade is needed. Fig. 4 shows the voltage and current for a $1.5 \mathrm{sec}$ cycle.

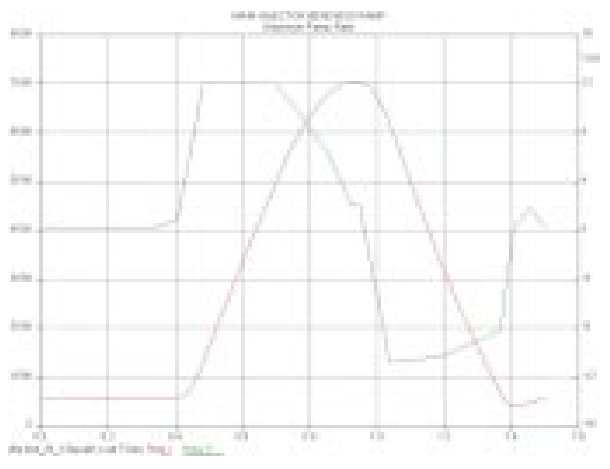

Figure 4: Magnet power supply voltage (green) and current (red).

\section{Large Aperture Quadrupoles}

In the Main Injector, a physical aperture bottleneck is at the quadrupoles upstream of the Lambertson magnets in several straight sections: MI 10, 30, 40, 52, 60 and 62. In order to reduce beam loss at these locations, large aperture quads need to be installed replacing the regular quads. The aperture will be increased from $83.48 \mathrm{~mm}$ to 102.24 $\mathrm{mm}$, i.e., 4 inches.

\section{Gamma-t Jump System}

Presently there is no $\gamma_{\mathrm{T}}$-jump system in the machine. But this system will be necessary in the upgrade. A description of this system can be found in Ref. [3]. It is a so-called first order jump system, making use of the zerodispersion straight sections. Its main advantage is small and localized perturbation to the lattice when the $\gamma_{\mathrm{T}}$-jump quads are activated. The system consists of 8 sets of pulsed quadrupole triplets. Each triplet has two quads in the arc and one of twice integrated strength in the straight section, with a phase advance of $\pi$ between each quadrupole. The power supply uses a GTO as the fast switch and a resonant circuit with a $1 \mathrm{kHz}$ resonant frequency. The beam pipe is made of Inconel 718.

This system can provide a $\Delta \gamma_{\mathrm{T}}$ from +1 to -1 within 0.5 ms. It gives a jump rate of $40001 / \mathrm{s}$, about 17 times faster than the normal ramp rate. Fig. 5 shows the quad current during the jump. The amplitude is adjustable. Fig. 6 shows the layout of the 8 triplets around the ring.

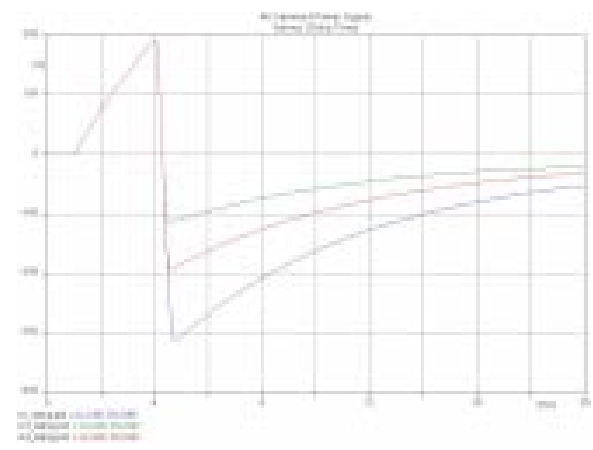

Figure 5: Magnet current of the pulsed $\gamma_{\mathrm{T}^{-} \text {-jump quads. }}$

*Work supported by the Universities Research Association, Inc. under contract No. DE-AC02-76CH03000 with the U.S. Dept. of Energy. \#chou@ fnal.gov 


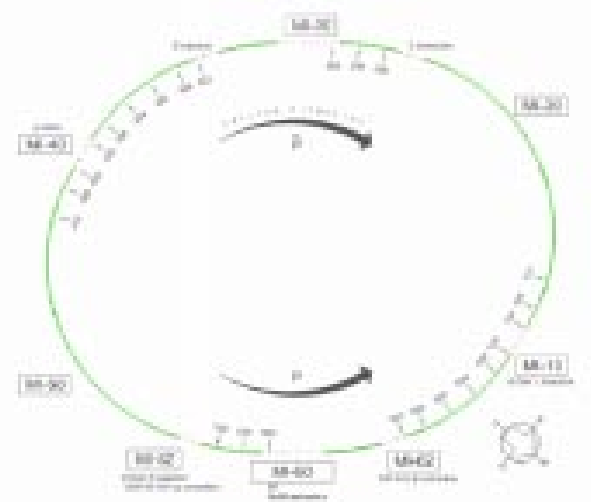

Figure 6: Layout of $8 \gamma_{\mathrm{T}}$-jump triplets. Green indicates the arcs, white the straight sections.

\section{Radiation Shielding and Collimation}

The shielding of the MI appears to be adequate for the upgrade. However, a collimation system is required in order to minimize the uncontrolled beam loss in the machine for reducing residual radioactivity so that handson maintenance can be performed. This system will be installed in MI 30. Fig. 7 illustrates a collimator.

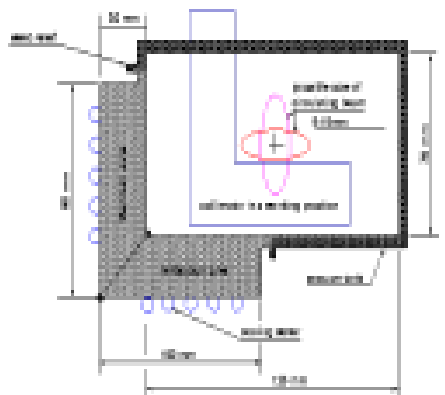

Figure 7: Secondary collimator cross-section.

\section{Kickers}

The MI beam pipe has a vertical aperture of 2-inch everywhere except at the kickers, which is 1.3-inch. In order to eliminate this bottleneck, the kicker aperture will be enlarged. Some kicker magnets also need to be rebuilt.

\section{Abort System}

With a modest upgrade, the present beam dump at MI 40 can absorb five times more protons. Fig. 8 shows the temperature rise of the abort dump.

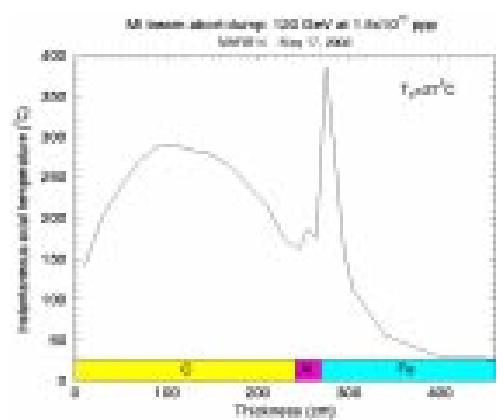

Figure 8: Maximum instantaneous temperature on the beam axis in the abort dump simulated by MARS.

\section{Passive damper and active feedback}

To suppress coupled bunch instabilities, both a passive damper and active feedback are needed. The former places nonlinear lossy materials (e.g., a special ferrite of which the loss parameter $\mu$ " is frequency dependent) in the RF cavity to damp the higher order modes (HOMs) while leaving the fundamental mode unaffected. The present longitudinal and transverse feedback systems can be used but need improvement.

\section{Mechanical and Utility}

The cooling capacity for magnets and power supplies appears to be sufficient in this upgrade. But the cooling system capacity for the RF system and cavities need to be doubled.

\section{NuMI Beam Line}

A $\$ 110 \mathrm{M}$ beam line for the neutrino experiment NuMI is under construction at Fermilab. It will use the protons from the MI. An upgraded MI would greatly enhance the physics potential of NuMI. To cope with $2 \mathrm{MW}$ beam power, the shielding and cooling in the target hall, decay pipe and hadron absorber need to be upgraded. The ground water problem is a concern but there is a reasonable solution (see Ch. 21 of Ref. [1]).

\section{COST ESTIMATE}

The cost estimate was done using a bottom-up method. Namely, the cost of each system upgrade was estimated by the engineers in the corresponding departments. The total upgrade cost is about $\$ 36 \mathrm{M}$, including EDIA.

There are two possible ways to implement the MI upgrade. It can be done as a single "all-included" Fermilab project. Or the upgrade can be accomplished through a series of accelerator improvement projects (AIPs).

\section{ACKNOWLEDGEMENT}

A group of accelerator physicists and engineers from Fermilab's Beams Division, Technical Division, and FESS and ES\&H sections contributed to this study. This paper is a report on behalf of this study group. The author expresses his thanks to them for their commitment and contributions to this study.

\section{REFERENCES}

[1] "Proton Driver Study II, Part 1," edited by G.W. Foster, W. Chou and E. Malamud, FERMILAB-TM2169 (May 2002). Also on the web: http://wwwbd.fnal.gov/pdriver/8GEV/

[2] W. Chou, "Intensity limitations in Fermilab Main Injector," PAC 1997 Proceedings, pp. 991-993.

[3] W. Chou et al., "Design of a gamma-t jump system for Fermilab Main Injector," PAC 1997 Proceedings, pp. 994-996. 